\title{
Alterstice
}

Revue internationale de la recherche interculturelle

International Journal of Intercultural Research

Revista International de la Investigacion Intercultural

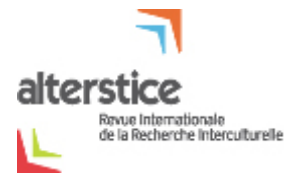

\section{Enseignants non immigrants et enseignants immigrants : convergences et divergences autour de la relation entre école et familles immigrantes}

\section{Malanga-Georges Liboy et Paulin Mulatris}

Volume 6, numéro 1, 2016

Prendre en compte la diversité à l'école

URI : https://id.erudit.org/iderudit/1038282ar

DOI : https://doi.org/10.7202/1038282ar

Aller au sommaire du numéro

Éditeur(s)

Alterstice

ISSN

1923-919X (numérique)

Découvrir la revue

Citer cet article

Liboy, M.-G. \& Mulatris, P. (2016). Enseignants non immigrants et enseignants immigrants : convergences et divergences autour de la relation entre école et familles immigrantes. Alterstice, 6(1), 91-103. https://doi.org/10.7202/1038282ar

\section{Résumé de l'article}

En tenant compte du défi de la communication école-famille, cette étude tente d'identifier les solutions esquissées par les professionnels de l'enseignement issus des communautés culturelles et de leurs collègues canadiens en Alberta. Les propos des participants et participantes sont analysés en s'inspirant du modèle écosystémique de Bronfenbrenner. À partir de quelques divergences et convergences observées dans les opinions des deux groupes d'enseignants, quelques pistes de solution qui amélioreraient la communication entre l'école et les familles immigrantes sont discutées. 


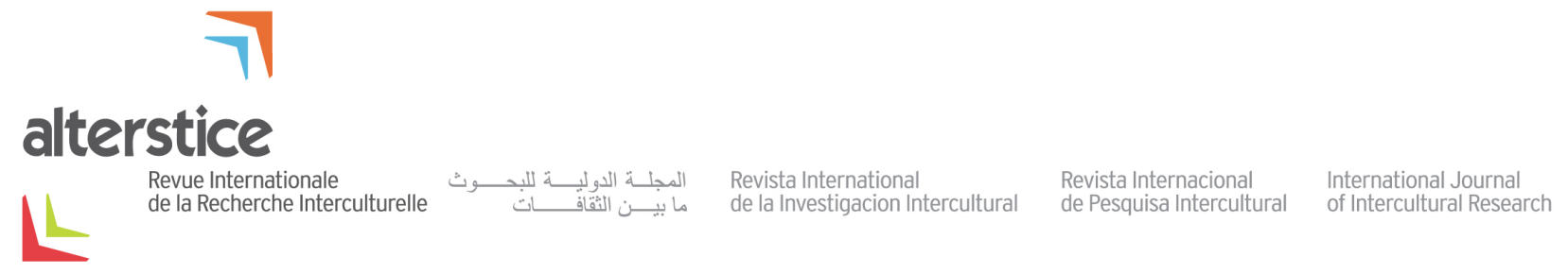

ARTICLE THÉMATIQUE

\section{Enseignants non immigrants et enseignants immigrants : convergences et divergences autour de la relation entre école et familles immigrantes}

Malanga-Georges Liboy ${ }^{1}$ et Paulin Mulatris ${ }^{2}$

\section{Résumé}

En tenant compte du défi de la communication école-famille, cette étude tente d'identifier les solutions esquissées par les professionnels de l'enseignement issus des communautés culturelles et de leurs collègues canadiens en Alberta. Les propos des participants et participantes sont analysés en s'inspirant du modèle écosystémique de Bronfenbrenner. À partir dequelques divergences et convergences observées dans les opinions des deux groupes d'enseignants, quelques pistes de solution qui amélioreraient la communication entre l'école et les familles immigrantes sont discutées.

\section{Rattachement des auteurs}

${ }^{1}$ Université Sainte-Anne, Pointe-de-I'Église, Canada ${ }^{2}$;Université de l’Alberta, Edmonton, Canada

\section{Correspondance}

paulin.mulatris@ualberta.ca

\section{Mots clés}

enseignants, immigrants, Canadiens, éducation, enfants, parents immigrants

\section{Pour citer cet article}

Liboy, G.-M. et Mulatris, P. (2016). Enseignants non immigrants et enseignants immigrants : convergences et divergences autour de la relation entre école et familles immigrantes. Alterstice, 6(1), 91-104. 


\section{Introduction et contexte}

Cet article approfondit certaines dimensions d'une étude plus vaste dont l'objectif général était de chercher à identifier les facteurs propices à une communication efficace entre enseignants et parents immigrants africains (Liboy, 2009). Partant du contexte albertain et considérant la diversité culturelle comme un facteur déterminant du milieu professionnel enseignant, nous nous intéressons ici aux solutions envisagées par les enseignants en vue d'améliorer la communication entre l'école et la famille et de contribuer à la réussite scolaire des jeunes.

L'Alberta est l'une des provinces canadiennes ayant connu un essor économique remarquable au cours des dernières années (Houle, Pereira et Corbeil, 2014; Statistique Canada, 2006). Cette situation a attiré des flux migratoires très considérables tant au niveau national qu'international, plus particulièrement entre 2005 et 2011. En 2006, la province a accueilli 9210 francophones, enregistrant ainsi une croissance démographique trois fois supérieure $(1,12 \%)$ à la moyenne nationale $(0,33 \%)$ en une seule année (Statistique Canada, 2006). Les deux grandes villes albertaines, Calgary et Edmonton, ont reçu respectivement 57940 et 31910 personnes. Sur une période de 20 ans (1991 et 2011), ces changements ont contribué à modifier la part relative de la population immigrante francophone dans l'ensemble de la population francophone, de $9 \%$ à 17,5\% (Houle, Pereira et Corbeil, 2014). En 2011, Edmonton et Calgary comptaient 10600 immigrants francophones, avec un léger avantage pour Calgary, dont le pourcentage d'immigrants francophones est passé de $16 \%$ à $25 \%$ (Houle, Pereira et Corbeil, 2014). Parmi ces nouveaux arrivés, on compte plusieurs familles longtemps restées au chômage dans d'autres provinces canadiennes, venues en Alberta pour tenter de trouver un emploi et de sortir de la précarité professionnelle (Hyman et Guruge, 2002).

S'agissant de la composition démographique de cette nouvelle population francophone albertaine, Houle, Pereira et Corbeil (2014) notent une proportion importante de minorités visibles (60\%) et, parmi ceux-ci, les Noirs seraient les plus nombreux en Alberta et dans les Prairies. S'agissant de la structure par âge de ces immigrants francophones, le groupe des 0 à 19 ans a connu une grande augmentation (de $12 \%$ à $15 \%$ ). L'étude évoquée indique que "la population immigrée francophone apparaît comme une population plutôt jeune (caractérisée par une proportion importante des 0-19 ans) en comparaison de celle des immigrants non francophones " (Houle, Pereira et Corbeil, 2014, p. 47). Ces flux migratoires ont modifié le paysage scolaire albertain. N'ayant pas anticipé les problèmes relatifs à l'intégration scolaire et sociale des élèves immigrants, plusieurs établissements scolaires font face à des défis difficiles à relever rapidement, tel le décalage des niveaux scolaires entre les élèves immigrés et les autres, des valeurs culturelles distantes de celles de la société d'accueil, une méconnaissance de la langue enseignée à l'école, une faible collaboration entre l'école et les familles immigrantes, etc. La majorité des conseils scolaires de la province n'ont pas eu d'autres choix que de s'ajuster aux effets découlant de ces changements démographiques. Le Conseil scolaire Centre-Nord (2006), le plus grand du réseau francophone à Edmonton, a vu depuis 2006 sa population estudiantine croître de $30 \%$ en trois ans, et cette tendance ne faiblit pas. En 2007-2008, par exemple, à Edmonton, l'école Sainte-Jeanne-d'Arc a vu sa population s'accroître de $30 \%$, Notre-Dame de $22 \%$, Père-Lacombe de $18 \%$, Gabrielle-Roy de $16 \%$, La Prairie (Red-Deer) de $25 \%$, Boréal (Fort-Mc Murray) de $20 \%$ (Conseil scolaire Centre-Nord, 2006). Cette croissance a eu des impacts tant sur le plan des infrastructures scolaires que du personnel enseignant. Pour remédier à cette situation, conformément aux recommandations du Rapport Caron (2008), les autorités scolaires ont mis en place un programme d'investissement rapide.

L'autre défi était de recruter un personnel enseignant francophone. Le Conseil scolaire a embauché près de 49 nouveaux enseignants de différentes origines ethniques pour pourvoir les postes vacants (Viens, 2009). Le milieu scolaire, jadis composé d'une population plutôt homogène sur le plan ethnoculturel, accueille aujourd'hui une clientèle étudiante diversifiée, des parents d'origines diverses et des enseignants issus de minorités ethnoculturelles. Ce changement répond aux vœux de certains parents immigrants, qui souhaitaient que "les écoles tiennent compte de la représentativité au sein du personnel enseignant et engagent des enseignants immigrants » (Liboy et Venet, 2011, p. 165). Plus récemment, cette préoccupation insuffisamment prise en compte a provoqué des débats communautaires, incitant le Conseil scolaire Centre-Nord (CSCN) à développer des politiques plus ouvertes dans l'embauche de son personnel (Bayon, 2015). 


\section{Une situation paradoxale}

L'école française en milieu minoritaire a été créée grâce à l'article 23 de la Charte canadienne des droits et libertés. Elle est destinée principalement aux ayants droit, c'est-à-dire aux Canadiens dont le français est la langue maternelle. Dès sa création, elle a été utilisée comme une institution de production identitaire et de survivance pour les communautés minoritaires francophones, qui ont vécu l'hostilité de la population majoritaire anglophone du pays (Faucher, 2002). Du fait de leur statut particulier, ces établissements scolaires ne sont pas tenus accepter les élèves immigrants "francophiles ». Une telle situation est susceptible de favoriser la délégitimisation du pluralisme francophone et interdirait aux immigrants francophones de se prévaloir des services de langue française pour leurs enfants (Daley, 2002). Par ailleurs, pour survivre, ces écoles doivent justifier leur pertinence du point de vue démographique, en ayant un nombre raisonnable d'élèves inscrits. À cause de certains facteurs (assimilation des jeunes, faible taux de natalité, vieillissement de la population, accroissement de la population immigrante francophone), ce type d'école s'est transformé et a ouvert ses portes aux autres francophones, ceux issus de l'immigration internationale ou d'autres provinces canadiennes (Brihmi, 2009). L'arrivée de jeunes immigrants a freiné la chute du nombre d'élèves et a contribué à la croissance significative de la population scolaire.

\section{La collaboration école-famille : une réalité complexe}

Les difficultés de collaboration entre les familles immigrantes et l'école des pays d'accueil sont soulignées dans la littérature (Akkari, 2000 et 2002). Les chercheurs reconnaissent que cette collaboration est déterminante dans le processus d'éducation d'un enfant (Kanouté, Vatz-Laaroussi, Rachédi et Doffouchi, 2008). Elle comporte des avantages pour les parents, les élèves, les enseignants, l'école et la communauté toute entière (Conseil supérieur de l'éducation, 1994). Une saine collaboration favorise de meilleures attitudes et un meilleur rendement scolaire de la part des élèves (Benoît, Rousseau, Ngirumpatse et Lacroix, 2008). Elle semble pourtant être compliquée dans le cas de certaines familles immigrantes dont la trajectoire migratoire est particulière et dont l'expérience de vie est marquée par des carences et des pertes (Benoît et coll. 2008). Ces familles rencontreraient des difficultés dues au fait que leurs valeurs culturelles et religieuses diffèrent de celles des communautés d'accueil. Elles connaîtraient aussi des barrières linguistiques ou une situation socioéconomique précaire et des problèmes d'isolement et de discrimination (Vatz Laaroussi, 2001). En 2003, par exemple, environ $35 \%$ des parents nouvellement arrivés en Alberta ne parlaient pas anglais, contrairement à leurs enfants dont le répertoire linguistique était plus large. La méconnaissance du système scolaire et des valeurs de la société d'accueil, la difficile intégration dans la communauté d'accueil, la ghettoïsation font que plusieurs de ces enfants se retrouvent en difficulté sur le plan scolaire (Bitupu, 2000 ; Godbout, 1989). La situation n'est pas plus facile pour le personnel enseignant qui déplore le manque de formation à l'éducation interculturelle, la faible collaboration avec les parents immigrants, l'insuffisance d'informations sur le parcours de vie des élèves et l'insuffisance des moyens d'interventions (Kanu, 2009).

\section{Le modèle écosystémique de Bronfenbrenner}

Comme nous l'avons mentionné, nous privilégions dans cet article le point de vue des enseignants, sachant que ceux-ci sont eux-mêmes pris dans des enjeux de diversité au sein de leurs propres relations professionnelles. Deux questions ont orienté notre analyse : 1) Quels sont, du point de vue des enseignants, les facteurs facilitant une communication efficace avec les parents immigrants? 2) Quelles sont les représentations des enseignants immigrants et non immigrants par rapport à la relation entre école et familles immigrantes ?

À la suite de Beckman (2003), nous nous appuyons sur la théorie écosystémique de Bronfenbrenner (1979; voir aussi Bronfenbrenner et Moris, 1998) pour analyser les solutions proposées par les participants à cette étude. Selon Bronfenbrenner (1979), en effet, le développement humain résulte des multiples interactions ayant lieu dès le départ entre l'enfant et les personnes qui l'entourent. Ces interactions se dérouleraient dans différents systèmes ou résulteraient de l'influence d'un système sur un autre: 1) le microsystème (qui entoure immédiatement l'enfant), 2) le mésosystème (formé d'interactions entre différents microsystèmes), 3) l'exosystème (formé d'interactions entre l'un des microsystèmes de l'enfant et un système qui lui est extérieur) et 4) le macrosystème (qui englobe la culture et les valeurs avec lesquelles l'enfant est en contact plus ou moins direct, par l'intermédiaire 
des différents autres systèmes). Ainsi les défis à l'intégration socioscolaire auxquels les élèves immigrants (et leurs parents) font face se situeraient à différents niveaux de ce modèle, et les solutions envisagées en dépendraient.

Bien que, plus récemment, ce modèle théorique ait été étendu aux processus proximaux (Tudge, Mokrova, Hatfield et Karnik, 2009), nous en retiendrons ici l'aspect systémique, dans la mesure où il nous permettra de classifier les différentes propositions des enseignants rencontrés en fonction des personnes ou instances susceptibles de les mettre en œuvre. Nous soulignerons également dans quelle mesure les solutions envisagées seraient bénéfiques pour les enfants.

\section{Méthodologie}

La méthodologie adoptée dans cette étude est qualitative et exploratoire, visant à combler un vide dans les écrits (Van der Maren, 1996). Notre approche prospective vise à identifier la façon dont les enseignants envisagent de favoriser la communication entre eux ainsi qu'avec les parents de leurs élèves issus des communautés ethniques.

\section{Profil des participants à l'étude}

Les participants à l'étude (Liboy, 2009) ont été recrutés, en 2008, dans des écoles de deux conseils scolaires (le Conseil scolaire Centre-Nord et le Edmonton Catholic Schools Board), par le biais des directeurs et directrices des écoles. Le pourcentage d'élèves issus de communautés ethniques dans ces écoles variait de $10 \%$ à plus de $90 \%$. Le tableau 1 présente le profil des participants ${ }^{1}$.

Tableau 1 : Profil des participants aux groupes de discussion

\begin{tabular}{|c|c|c|}
\hline Caractéristiques & Parents & Personnel enseignant \\
\hline Nombre & 10 & 12 \\
\hline Pays d'origine & $\begin{array}{l}\text { Afrique : Algérie (1), Côte d'Ivoire } \\
\text { (1), Mauritanie (1), République } \\
\text { démocratique du Congo (6), } \\
\text { Rwanda (1) }\end{array}$ & $\begin{array}{l}\text { Provinces canadiennes: Québec (5), } \\
\text { Alberta (2), Nouveau-Brunswick (1). } \\
\text { Afrique : Rwanda (1), République du } \\
\text { Congo (1), République démocratique du } \\
\text { Congo (2) }\end{array}$ \\
\hline Hommes & 8 & 8 \\
\hline Femmes & 2 & 4 \\
\hline Immigrants & 10 & 4 \\
\hline Non-immigrants & - & 8 \\
\hline $\begin{array}{l}\text { Programme scolaire } \\
\text { francophone } \\
\text { anglophone }\end{array}$ & & $\begin{array}{l}8 \\
4\end{array}$ \\
\hline
\end{tabular}

Même si les parents immigrants ont participé à cette recherche, nous ciblons dans cet article les perceptions des enseignants.

\section{Procédure et instrument de collecte de données}

Nous avons eu recours à trois groupes de discussion pour recueillir les perspectives de participants. Legendre (2005) définit le groupe de discussion comme un groupe de personnes réunies dans un même endroit afin de débattre de thèmes communs ou proposés par un animateur. Nous avons utilisé cette technique parce qu'elle mise sur la dynamique du groupe sans viser à imposer un consensus ni même à faire valoir un point de vue particulier (Patriciu, 2001). Les trois groupes réunissaient respectivement le personnel enseignant, les parents puis les parents

\footnotetext{
${ }^{1}$ Dans cette étude, selon le contexte canadien, nous entendons par immigrant les personnes venant directement de l'étranger (Europe, Afrique, Amérique, Asie, etc.) admises au Canada comme résidents permanents ou comme réfugiés. Les personnes réinstallées en Alberta et arrivant d'autres provinces canadiennes (Québec, Ontario, etc.) sont considérées comme des migrants internes.
} 
et les enseignants ensemble, au Campus Saint-Jean (faculté francophone de l'Université d'Alberta, Edmonton). Chacune des discussions a duré entre une heure et une heure et demie. Un guide d'entretien et une vidéo de mise en situation ont été utilisés pour orienter ces entretiens de groupe. La validation des données recueillies a été progressive. Les éléments émergeant du premier groupe de discussion ont été validés dans le deuxième, puis nous nous sommes servis de la troisième discussion pour valider les éléments des deux premiers groupes. Enfin, une validation globale de toutes les données recueillies a été faite auprès de certains participants, qui ont été consultés en vue d'une approbation.

Les objectifs, les questions, les différents documents consultés ainsi que l'approche méthodologique qualitative et le cadre conceptuel adoptés ont orienté cette étude vers une analyse de contenu de type thématique (Liboy, 2009). La démarche a consisté à organiser les discours du groupe de discussion puis à démembrer le texte en petites unités de sens et à procéder à l'attribution de codes tout en restant fidèle au message émis (Paillé, 1995). Après la thématisation effectuée à l'intérieur du groupe de discussion, une combinaison de différents résultats a été réalisée pour clore la démarche. Nous avons regroupé les propos des participants selon que les solutions étaient susceptibles d'émaner du mésosystème famille-école, des exosystèmes enseignants-conseil scolaire et parentsemployeurs ou du macrosystème en cause dans les relations entre les familles immigrantes et l'école canadienne, puisque les valeurs et les cultures des personnes immigrantes sont souvent perçues comme différentes des valeurs et de la culture canadiennes.

\section{Résultats}

\section{Le mésosystème}

Le mésosystème permet de faire émerger les liens, les relations et les interactions qui existent entre les deux microsystèmes (école et famille) où se retrouve l'enfant issu de l'immigration, qui est au centre même de cette étude, le but ultime étant de favoriser sa réussite scolaire.

\section{Les responsabilités des familles immigrantes}

Si les enseignants (immigrés ou non) considèrent avoir la responsabilité de travailler avec les familles afin d'améliorer les résultats scolaires des élèves, il n'en demeure pas moins, selon eux, que les familles sont coresponsables de la réussite de leurs enfants. Les familles immigrantes doivent s'efforcer de venir les rencontrer malgré leurs occupations. Au-delà de la multitude d'outils qu'on peut leur offrir, elles doivent essayer de comprendre le fonctionnement du système scolaire :

Il faut aussi, quand même, que ces personnes-là [les parents immigrants] s'aident; tu comprends, leur volonté de venir s'informer, apprendre par rapport au système. (Enseignant non immigrant)

Les enseignants sont conscients des contraintes ("de travail, familles, de survie ») auxquelles ces parents sont soumis. Sachant que la majorité des établissements scolaires n'organisent pas de séances d'information, une enseignante immigrante estime qu'il faudrait régler le problème lié au peu d'information dont disposent les familles avant de les responsabiliser sur leur rôle éducatif :

Les parents doivent savoir qu'ici la responsabilité est partagée. Il faudrait les en aviser lorsqu'ils viennent inscrire leur enfant à l'école, leur dire : "Bon, madame, monsieur, ici comme vous voyez ce n'est pas seulement nous qui allons éduquer votre enfant à l'école, mais c'est une responsabilité partagée. Dans la mesure du possible, on va vous demander de vous impliquer dans l'éducation de votre enfant ». (Enseignante immigrante)

Si, dans la vision canadienne de l'éducation, la responsabilité des familles dans la réussite des enfants est une évidence, ce n'est pas forcément le cas des familles immigrantes, qui viennent de pays où les rôles des familles et de l'école sont distincts. Il en ressort également que les enseignants non immigrants ne sont pas forcément au courant de cette situation, et qu'il leur est peut-être difficile de comprendre l'attitude des familles immigrantes. Ce n'est pas le cas pour les enseignants immigrants, qui connaissent cette réalité depuis leur pays d’origine. 


\section{Visites au domicile des familles immigrantes}

Il n'est pas habituel pour un professionnel de l'enseignement au Canada de rendre visite aux familles de ses élèves. Certains estiment qu'ils n'ont pas de temps pour le faire, d'autres hésitent à mélanger les rôles et les responsabilités des parents et du personnel enseignant. Par contre, nombre de parents immigrants participant à cette étude sont ouverts à l'idée d'accueillir les enseignants ou d'autres intervenants chez eux. Certains enseignants immigrants qui l'ont déjà fait se sont dits interpellés comme immigrants d'agir ainsi auprès de ces familles afin de les convaincre de s'impliquer davantage dans le processus d'enseignement de leurs enfants.

Le peu de fois que j'ai fait ça, ça a marché à $100 \%$ et puis ça a diminué le problème de discipline avec les enfants. Les enfants m'ont fait confiance, ils me traitent comme leur tante à l'école. Pourtant, on n'a rien comme lien, et puis à chaque fois je me dis : «le jour où je ne serai pas là ? » Parce que le fait de me voir venir là, d'abord ils vont venir me saluer avec des tonnes d'amour [...] et les petites choses [les petites bêtises] qu'ils étaient en train de faire ils ne les font pas, pourquoi ? Ils m'ont vue chez eux. (Enseignante immigrante)

Pour les parents participants, l'envoi de messages aux parents immigrants ne suffit pas pour inciter ces derniers à échanger avec l'école. Ils ne sont pas habitués à répondre à de tels messages, alors qu'une visite à domicile, en créant la proximité, peut changer cette attitude et améliorer la communication entre l'école et les familles immigrantes. Comprenant les difficultés des familles immigrantes nouvellement arrivées, le personnel enseignant surtout immigrant - reconnaît la nécessité de trouver des voies et moyens d'entrer en interaction sur une base régulière avec ces parents, malgré le manque de temps.

Les familles immigrantes ne sont pas souvent à l'école, elles ne participent pas à la vie de l'école de leurs enfants [...]. Elles sont occupées à nouer les deux bouts et ce n'est pas très évident pour elles. Je pense qu'il faudrait chercher le moyen de rejoindre ces gens-là malgré l'emploi du temps chargé. Je comprends en tant qu'enseignante que nous aussi de notre côté, on n'a pas beaucoup de temps, mais il faut chercher des stratégies pour rejoindre les parents des familles immigrantes. (Enseignante immigrante)

Même si les visites à domicile des familles des élèves ne sont pas répandues dans le système scolaire canadien, les enseignants immigrants souhaiteraient voir leurs collègues non immigrants s'intéresser aux problèmes qui se passent dans ces familles afin de bien les comprendre et de tisser un lien de collaboration solide.

\section{Attentes et compétences des familles immigrantes}

On reproche aussi bien aux familles d'origine canadienne, notamment de milieu défavorisé, qu'aux familles étrangères de ne pas avoir d'attentes précises à l'égard de l'école. À cause de leur sentiment d'impuissance face aux institutions, elles s'en remettent au personnel enseignant, auquel elles accordent leur confiance. Les enseignants réalisent que ces parents, parfois considérés comme "irresponsables", ne connaissent pas les attentes de l'école et, comme ils entretiennent des relations superficielles avec les enseignants de leurs enfants, ils ne seront jamais informés de ce que les enseignants attendent d'eux. Certains enseignants non immigrants croient qu'il est plus difficile de communiquer avec les familles immigrantes nouvellement arrivées en Alberta qu'avec les autres familles car elles ont peu d'attentes pour leurs enfants et ne comprennent pas celles des enseignants. De plus, la distance entre les valeurs culturelles de ces familles et celles de l'école est grande.

Il y a un manque de communication. Ici, ce n'est pas un manque de volonté, je pense que c'est juste qu'il faut prendre le temps peut-être de trouver du temps de se rencontrer pour se parler et se dire: plus de rencontres informelles, plus de proximité, voici mes attentes parce que le programme de l'Alberta c'est ceci-cela et voici comment ça fonctionne. Je ne sais pas si c'est comme ça pour vous, mais je n'entends rien des parents [immigrants]. (Enseignante non immigrante)

Ce point de vue n'est pas partagé par les enseignants immigrants, qui croient que l'enseignant a le devoir d'informer les parents immigrants sur le fonctionnement du système canadien et ses attentes.

Je pense que nous, enseignants, avons le mandat [...] d'éduquer les parents aussi, puisqu'on est en contact avec les parents plus que les autres personnes. Quand j'ai un problème avec un élève, la première personne que je contacte, c'est le parent [...] nous qui venons de l'Afrique, avons été élevés tels que c'est l'école qui s'occupait de nous, pas les parents. À la fin de 
l'année scolaire, ils regardent le bulletin : il est passé ou il n'est pas passé. Comment ça s'est passé le long de l'année ? lls ne savent pas. Alors, c'est à nous d'expliquer comment fonctionne l'école au Canada. (Enseignant immigrant)

Les compétences éducatives des familles varient selon le niveau d'instruction et les disponibilités des parents. Les types d'intervention proposés sont divergents. Deslandes et Bertrand (2004) affirment que les parents s'impliquent dans l'éducation à condition d'y être motivés et que cette motivation est directement proportionnelle à leur sentiment d'être compétents pour le faire et d'avoir une influence sur la réussite scolaire de leurs enfants. En ce qui concerne le degré d'implication des parents, nous avons relevé une divergence entre les représentations des enseignants non immigrants, qui croient que ces parents devraient agir comme les autres parents non immigrants pour aider leurs enfants dans leur apprentissage, et les enseignants immigrants, qui insistent sur l'assistance à ces familles pour les amener à s'y engager.

\section{Besoins linguistiques des familles immigrantes et messages dans les langues étrangères}

Il est difficile d'établir une communication efficace entre les familles immigrantes et l'école si l'on ignore les problèmes de cette clientèle. Il est indispensable, selon le personnel enseignant, de résoudre cette question dès le début de l'année scolaire. Lorsque les parents viennent pour inscrire leurs enfants, l'administration scolaire devrait vérifier si ceux-ci parlent et comprennent la langue d'usage à l'école.

Lorsque les élèves viennent pour s'inscrire, à ce moment, il faut s'assurer pouvoir communiquer avec eux. (Enseignant non immigrant)

Si les parents rencontrent des difficultés à communiquer, l'école devrait mettre en place des stratégies de communication adaptées en accord avec eux afin de pouvoir établir des contacts permanents. La communication écrite devrait également tenir compte des besoins linguistiques des familles immigrantes pour que s'établisse la communication entre elles et l'enseignant. Une telle initiative constituerait un moyen efficace pour atteindre plusieurs familles qui éprouvent de la difficulté à communiquer en français et en anglais. Pour certains enseignants non immigrants, une telle proposition est pertinente, mais son application peut s'avérer difficile, voire impossible dans toutes les écoles à cause de l'insuffisance des ressources tant humaines que financières dont disposent les conseils scolaires et de la masse critique d'élèves concernés.

Je veux dire: je suis d'accord si on a la personne, comme un enseignant qui parle français et qui parle swahili, qui se débrouille bien en anglais, qui peut enseigner, c'est magnifique; mais sincèrement ce n'est pas toujours facile [...]. (Enseignant non immigrant)

La discussion qui a eu lieu autour de la possibilité de traduire les messages en différentes langues souligne une fois encore qu'il ne sera pas facile de satisfaire ces besoins pourtant criants. Il est difficile de savoir s'il s'agit d'un problème d'ordre pratique et financier ou plutôt associé à la lente évolution des mentalités des organismes et institutions concernés.

\section{Diversification des canaux de communication}

La communication des enseignants avec les familles immigrantes se fait à plusieurs reprises dans l'année scolaire. Plusieurs moyens sont utilisés pour joindre les parents d'élèves (messages sur papier, électroniques ou téléphoniques, rencontres...). Si certains parents sont faciles à rejoindre, d'autres le sont moins, et c'est le cas des familles immigrantes nouvellement arrivées en Alberta. Les participants à cette étude ont suggéré quelques pistes à suivre relativement aux canaux de communication les plus susceptibles de faciliter les contacts avec les familles immigrantes. II ne faut pas perdre de vue que ces familles ont des besoins particuliers et des valeurs culturelles d'origine différentes de celles des familles non immigrantes. Certaines des propositions semblent ne pas être conformes aux normes de communication acceptées dans les écoles de la communauté d'accueil. Les participants à l'étude proposent que les informations qui font l'objet d'une communication entre l'école et les familles soient divisées en deux groupes: les informations d'ordre public (invitation à une réunion, annonce d'une activité parascolaire, etc.) et celles d'ordre privé (rendement scolaire de l'élève, ses difficultés ou comportements à l'école). 
Selon les enseignants, immigrants particulièrement, les informations d'ordre public emprunteraient des canaux généraux, ceux qui n'assurent pas de confidentialité. Compte tenu de la tradition orale, caractéristique de la plupart des pays d'origine de familles immigrantes, ces dernières souhaitent que les enseignants recourent davantage à la communication orale. Les messages passeraient rapidement de bouche à l'oreille, par le réseautage, par le jumelage ou par des contacts directs.

Je viens d'une tradition, d'une culture où la tradition orale est forte et, souvent même dans la communauté, quand on dit quelque chose à une personne, la personne transmet, et ça passe de bouche à oreille plus facilement que si c'était du papier, un communiqué ou quelque chose comme ça. Alors peut-être que ça serait une stratégie à utiliser. (Enseignante immigrante)

Le jumelage consiste à associer deux familles immigrantes (l'une ne parle pas la langue de l'école et l'autre oui) partageant la même origine et parlant la même langue, afin que l'un des parents, qui communique régulièrement avec l'enseignant, transmette les messages d'ordre général au parent qui ne la parle pas.

Moi, j'ai déjà fait ça dans ma classe. Je demandais aux parents : "Quand vous recevrez une information, je vous demande, si vous vouliez m'aider, de téléphoner à l'autre parent qui parle la même langue ». S'il s'agit d'une excursion, ces parents vont expliquer aux autres le lieu, si les enfants auront besoin des patins et autres besoins. (Enseignant non immigrant)

Selon une enquête menée auprès des familles nouvellement arrivées en Alberta, près de $69 \%$ des personnes interrogées recourraient au bouche-à-oreille pour accéder aux informations (Mulatris, 2008). Cette pratique semble être plus commode et efficace que des messages écrits envoyés à la fin de la journée par les enseignants. Malgré son efficacité, elle ne recueille pas l'adhésion de tous les enseignants, tant non immigrants qu'immigrants, surtout ceux qui tiennent au respect des normes déjà établies au Canada sur la confidentialité des informations personnelles.

Pour garantir la confidentialité des informations concernant les élèves, les enseignants proposent de recourir aux moyens de communication conventionnels, tels que le téléphone, les contacts directs avec les familles, les messages écrits dans l'agenda ou courriel, etc. D'autres enseignants souhaiteraient une responsabilisation de l'élève sur certains messages le concernant.

Mais si, l'école, à travers les élèves, peut envoyer les messages à la maison. Tout simplement parce que l'école ne peut répondre toute seule aux besoins de la multiplicité des cultures qui y existent. (Enseignant non immigrant)

Les propositions des enseignants font ressortir ici des éléments du modèle de communication de Jakobson (1963) et vont dans le même sens que plusieurs auteurs quant à la nécessité de recourir à des canaux de communication diversifiés pour contacter les familles immigrantes nouvellement arrivées. Nous avons noté une divergence entre les enseignants immigrants, largement favorables à ces propositions qui se révèlent conformes aux valeurs culturelles des familles immigrantes d'origine africaine, et les enseignants non immigrants, qui tiennent aux lois en vigueur et aux habitudes instaurées depuis des années dans la communication entre les écoles et les familles.

\section{L'exosystème : redéfinir la mission de l'école en milieu minoritaire}

L'exosystème est un système qui englobe les interrelations entre un contexte dont l'élève ne fait pas partie et un microsystème auquel il appartient. Dans le cas qui nous occupe, les structures et les organismes à caractère scolaire albertains sont fréquemment en interaction avec les écoles et les familles des élèves immigrants. Dans cette étude, le recours à l'exosystème et au macrosystème, en ce qui concerne les représentations des enseignants, est mineur comparativement au mésosystème, qui est au cœur de la recherche.

La mission de l'école francophone en milieu majoritairement anglophone, comme c'est le cas pour l'Alberta, est, selon l'article 23 de la Charte canadienne, de protéger la culture et la langue françaises. De plus en plus, ces écoles s'ouvrent aux élèves des familles immigrantes francophones et, du coup, cette situation change sa mission initiale. Celle-ci devrait être redéfinie en tenant compte de la présence de cette nouvelle clientèle. Un enseignant canadien 
approuve cette proposition et pense qu'il est temps que la société d'accueil agisse dans le sens du changement intervenu dans la communauté.

Maintenant, la communauté francophone n'est pas seulement [composée] des Francophones qui ont l'identité et leur langue à préserver pour faire face à l'assimilation autour d'eux. Ce n'est pas juste cette identité-là. Moi, je suis de cette communauté-là et puis on voit que la communauté francophone est beaucoup plus large, qu'il y a plusieurs cultures qui sont là. (Enseignant non immigrant)

Dans le but de répondre à cette question, cet enseignant a créé un comité d'accueil au sein de son école. L'objectif fixé est d'abord de créer un climat social favorable à l'accueil des nouveaux élèves d'origine étrangère et de les intégrer dans la vie scolaire.

C'est un comité qui cherche à changer, [non] pas l'ambiance - je ne veux pas dire qu'il n'y a pas d'ambiance à l'école -, mais il y a définitivement beaucoup de travail qu'on peut faire sur la façon dont on crée une ambiance plus accueillante pour tout le monde. (Enseignant non immigrant)

Deux constats se dégagent de ces pistes de solution. D’abord, l'école en milieu minoritaire francophone devrait reconnaître la présence de ces élèves, qui contribuent à la survie de ces institutions, et la spécificité des jeunes immigrants avec des besoins spéciaux. Ensuite, il est urgent d'intégrer en même temps leurs familles dans la vie de l'école. La mission de l'école francophone devrait faire l'objet de concertation entre la communauté d'accueil et les familles immigrantes francophones.

\section{Le macrosystème : vécu, valeurs et relation de confiance}

Certaines difficultés qui entravent la communication entre les enseignants et les parents immigrants prennent racine dans la distance qui sépare les valeurs culturelles des uns et des autres. La connaissance du vécu et des valeurs des familles pourrait avoir des répercussions positives, et notamment favoriser une relation de confiance entre le personnel enseignant et les parents. Les enseignants immigrants ont la facilité de bien cerner les difficultés qui engendrent le manque de communication avec les parents immigrants.

J'avais une excursion à faire avec mes élèves, dont des filles somaliennes qui sont musulmanes, mais leurs parents avaient refusé de signer la feuille de permission. J'ai compris que c'était des filles musulmanes et puis je ne suis pas musulmane, mais j'ai compris qu'ils avaient peur que leurs enfants aillent se coucher quelque part où ils ne sont pas là. Alors j'ai téléphoné aux parents. J'ai dit : "Pourquoi vous ne signez pas ? C'est quoi, votre problème ? » Ils ont dit : " Non, on ne veut pas laisser notre fille aller coucher ailleurs ». J'ai répondu: "Écoutez, je serai là ». - "Même si vous êtes là, ça ne suffit pas ». Alors, on a vraiment discuté et puis j'ai compris leur inquiétude. C'est qu'elle allait se dévoiler et allait prendre la douche avec une personne. J'ai accepté - j'ai demandé leur chambre - qu'elles allaient rester voilées, se laver sans qu'il y ait des jeunes qui soient là. (Enseignante immigrante)

La communication du vécu et des valeurs des parents pourrait aussi permettre d'éviter certains problèmes de communication auxquels il est relativement facile de remédier.

Une autre façon de communiquer : j'ai une amie qui m'a raconté que, dans sa classe, un des parents sait lire le message qui est écrit dans l'agenda, il le comprend, mais il ne veut pas lui répondre parce qu'il n'écrit pas bien. Puis il craint de se faire juger s'il n'écrit pas bien. [...] Moi, je croyais qu'il ne voulait pas communiquer avec moi, qu'il ne regardait pas mes messages. (Enseignante non immigrante)

Plus l'enseignant est en contact avec ces parents, plus il connaît leurs difficultés et plus il est en mesure de bien assister l'élève dans ses apprentissages. Les enseignants immigrants connaissent la situation de ces familles, comprennent très bien les barrières culturelles auxquelles elles font face. Ils sont capables de bien saisir les problèmes de ces parents, parce qu'ils ont vécu les mêmes expériences ou des expériences similaires. Ils font souvent un parallèle entre la situation des familles immigrantes et leur propre vécu, pour inciter les enseignants non immigrants à faire un pas vers ces familles. 


\section{Discussion}

Le personnel enseignant albertain rencontre des difficultés dans ses relations avec les familles immigrantes nouvellement arrivées. II est malgré cela animé par une volonté de les engager dans le processus éducatif de leurs enfants. Cependant, en comparant les discours des uns et des autres, nous observons que les enseignants immigrants sont plus proches des perceptions (valeurs, normes, cultures) des familles immigrantes. Cette situation s'explique, du moins, à notre avis, par le fait qu'ils ont des vécus et des trajectoires migratoires similaires (Vatz Laaroussi, 2007) et que certains sont eux aussi des parents qui, à leur arrivée au Canada, ont eu à vivre des difficultés semblables: refus de reconnaissance des acquis, méconnaissance du système scolaire, différences culturelles (Cook, 2009; Kanouté, Vatz-Laaroussi, Rachédi et Doffouchi, 2008; Lai et Huffey, 2009). Lors des échanges engagés pendant cette recherche, certains enseignants non immigrants ont appris, de leurs collègues immigrants, quelques informations en rapport avec la pédagogie ouverte et les différentes stratégies pour interagir avec les familles immigrantes. Les enseignants non immigrants ont proposé d'intensifier la collaboration entre collègues afin de mieux appréhender la problématique des familles immigrantes dans les écoles. Ils souhaitent recourir aux enseignants immigrants comme "personnes-ressources» dans leurs échanges avec les familles immigrantes. Le personnel enseignant partage également beaucoup d'opinions sur la question des facteurs susceptibles d'améliorer la communication entre les enseignants en général et avec les parents immigrants en particulier.

Nous avons observé des divergences de perceptions entre enseignants immigrants et non immigrants. Alors que les premiers soutiennent que les parents immigrants ont besoin d'être assistés pour comprendre le fonctionnement du système scolaire canadien avant de s'engager, les seconds considèrent que ces parents manquent à leur responsabilité parentale. De plus, lorsque les enseignants non immigrants évoquent le manque de moyens (budget, ressources humaines) et le respect des normes portant sur la confidentialité des informations, les enseignants immigrants encouragent la mise en place de nouvelles stratégies (communication orale, bouche-à-oreille, jumelage) pour rejoindre ces familles. Nonobstant ces quelques divergences, le personnel enseignant partage des points de vue similaires sur les relations interpersonnelles, les moyens de communication, les séances de formation et d'information, l'ouverture de l'école, l'accueil, la visite à domicile, etc.

\section{Conclusion}

Les problèmes relatifs à la défaillance de communication entre les enseignants et les parents immigrants sont bien connus. Plusieurs solutions pour les résoudre existent déjà mais ne produisent pas les effets attendus. Cette étude a permis d'entreprendre une consultation auprès des acteurs éducatifs concernés, et nous avons présenté pour cet article le point de vue des enseignants, afin d'explorer quelques pistes de solution. Les participants ont suggéré des solutions situées dans les différentes strates du modèle écologique, mais particulièrement dans la strate appelée " mésosystème ", car cette dernière s'intéresse plus aux interactions qui se passent entre l'école et les familles (Bronfenbrenner et Evans, 1998). Une meilleure compréhension du vécu et de la trajectoire des familles immigrantes par le personnel enseignant et les autres intervenants permettrait à ces derniers de prendre les dispositions qui s'imposent pour éviter des problèmes liés à l'incompréhension ou à la mésentente, et elle faciliterait l'ouverture du personnel enseignant aux familles immigrantes.

Les enseignants non immigrants ont réalisé l'importance d'avoir des collègues issus de milieux immigrants, capables d'intervenir auprès des familles immigrantes grâce à la similarité d'expériences sociales. Une bonne collaboration entre ces deux catégories d'enseignants pourrait améliorer la compréhension des problèmes des élèves, qu'ils appartiennent à la communauté d'accueil ou qu'ils soient issus de l'immigration. Cette situation favoriserait l'adaptation des pratiques pédagogiques et des programmes aux besoins spécifiques des jeunes.

Depuis quelques années, les écoles francophones accueillent de plus en plus d'élèves issus de l'immigration. Ces changements ne sont pas encore suivis par une formation adéquate des enseignants à l'éducation interculturelle. Cette étude réalisée en Alberta pourrait être étendue à l'ensemble du Canada, en tenant compte des caractéristiques propres à différents milieux francophones minoritaires. Elle pourrait améliorer les perceptions des enseignants non immigrants envers les familles immigrantes, la participation des parents immigrants à la vie de 
leur communauté et de l'école, le rendement scolaire des élèves immigrants et enfin favoriser l'attachement des familles immigrantes à la culture et à la langue françaises.

\section{Références bibliographiques}

Akkari, A. (2000). La gestion de la diversité culturelle dans le système éducatif fribourgeois. In F. Tanon et N. Chiasson, l'interculturalité en milieu culturellement homogène : un défi pour la formation professionnelle,7(3), p. 407-431.

Akkari, A. (2002). La gestion de la diversité culturelle dans le système éducatif fribourgeois, Cahiers de la recherche en éducation, 31, p. 71-79.

Bayon, A. (2015). La FRAP suscite enthousiasme et questionnements [en ligne]. Le Franco, avril. http://www.lefranco.ab.ca/francophonie/albertaine/item/1155-la-frap-suscite-enthousiasme-etquestionnements.html

Beckman, P. J. (2003). Relations parents et professionnels dans des structures éducatives inclusives. Éducation et francophonie, 31(1).

Ben Ayed, C. (2002). Relations familles/école. SNUIPP 89. Document téléaccessible à l'adresse http://89.snuipp.fr. Consulté le 13 mai 2005.

Benoît, D. (1995). Introduction aux sciences de l’information et de la communication. Paris : Les Éditions d'organisation.

Benoît, M., Rousseau, C., Ngirumpatse, P. et Lacroix, L. (2008). Relations parents immigrants-écoles dans l'espace montréalais : au- delà des tensions, la rencontre des rêves. Revuedes sciences de l'éducation,34(2), 313-332.

Bitupu, M. (2000). Défi d’intégration de la communauté congolaise dans la société canadienne. In C. Couture et J. Bergeron (Eds.), L'Alberta et le multiculturalisme francophone témoignages et problématiques (p. 111-127). Edmonton: Centre d'études canadiennes de la Faculté Saint-Jean.

Brihmi, M. (2008). L'incorporation citoyenne des membres des minorités ethniques francophones. L'express, 9, semaine du 4 au 10 mars, 2008.

Bronfenbrenner, U. (1979). The ecology of human development: Experiments by nature and design. Cambridge, MA : Harvard University Press.

Bronfenbrenner, U. et Morris, P. (1998). The ecology of developmental processes. In W. Damon and R.M. Lerner (Eds), Handbook of child psychology, vol. 1: Theoretical Models of Human Development. New York : Wiley, pp. 993-1028.

Bronfenbrenner, U. et Evans, P. (1998). Developmental science in the 21 st. Century: Emerging theoretical models, research designs, and empirical findings. Social Development, 9, 115-125.

Campey, J. (2002). Immigrant children in our classrooms: beyond ESL. Education Canada. (D9643-1).

Caron, D. (2008). Besoins en infrastructures de Conseil scolaire Centre-Nord, rapport conçu pour le CSCN.

Comeau, J. et Salomon, A. (1994). La participation à l'école : Une recherche de sens pour les intervenants. Laval : Agence d'Arcy.

Conseil scolaire Centre-Nord. (2006). Défis de l'éducation francophone à l'ère de la croissance en Alberta. Mémoire. Edmonton.

Conseil supérieur de l'éducation (1994). Être parent d'élève du primaire : Une tâche irremplaçable. Québec : Gouvernement du Québec.

Cook, D. (2009). Le nouvel Ouest : une crise identitaire imminente dans la ville du Stampede, Nos diverses cités, 6, 78-83, Printemps 2009. 
Dalley, P. (2002). Le multiculturalisme et l'école de la minorité francophone au Canada. In C. Couture et J. Bergeron (Éds), L'Alberta et le multiculturalisme francophone. Edmonton : Centre d'études canadiennes de la Faculté Saint-Jean et l'Association multiculturelle francophone de l'Alberta, 127-143.

Deslandes, R. et Bertrand, R. (2004). La recherche sur l'entrée à la maternelle, des résultats qui parlent... Revue préscolaire (AÉPQ),42 (1), 10-18.

Faucher, R. (2001). L'école française en milieu minoritaire. Revue Documentaire. Fédération canadienne des enseignants. www.circem.uottawa.ca/pdf/01-Revue_documentaire. Consulté le 07 mars 2008.

Gayet, D. (dir.) (1999). L'école contre les parents. Paris : INRP.

Godbout, G. (1989). La réalité multiculturelle des écoles. Dimensions, 1(1), 7-14.

Houle, H, Pereira, D. et Corbeil, J.P. (2014). Portrait statistique de la population immigrante de langue française à l'extérieur du Québec (1991 à 2011). Statistique Canada.

Hyman, I., et Guruge, S. (2002). A review of theory and health promotion strategies for new immigrant women. Canadian Journal of Public Health, 93(3), 183-187.

Jakobson, R. (1963). " Linguistique et poétique », Essais de linguistique générale. Paris : Minuit (p. 209-248).

Kanouté, F., Vatz-Laaroussi, M., Rachédi, L. et Doffouchi, M.-T. (2008). Familles et réussite scolaire d'élèves immigrants du secondaire. Revue des sciences de l'éducation, 34(2), 265-289.

Kanu, Y. (2009). Parcours scolaires favorisant l'intégration sociale des élèves réfugiés africains au Manitoba. Metropolis, 6 (printemps).

Lai, D.W. et Huffey,N. (2009). La discrimination vécue par les minorités visibles dans les petites collectivités, Nos diverses cités, 6, 136-141.

Legendre, R. (2005). Dictionnaire actuel de l'éducation (3è édition). Montréal : Guérin.

Liboy, M.-G. et Venet, M. (2011). Participation des parents immigrants à l'école en Alberta : point de vue des parents congolais, Journal of International Migration and Integration,12 (2), 155-171.

Liboy, M.-G. (2009). Étude des facteurs susceptibles de faciliter la communication entre l'école et les familles immigrantes en Alberta : Point de vue des parents immigrants et du personnel enseignant, Thèse de doctorat, Sherbrooke, Université de Sherbrooke.

Mulatris, P. (2008). Pour réussir un projet communautaire : une perspective immigrante. Dans P. Mulatris (dir.), L'intégration des immigrants francophones dans l'Ouest du Canada. (p.46-50). Actes du Colloque. Edmonton : Bibliothèque et Archives Canada.

Paillé, P. (1996). De l'analyse qualitative en général et de l'analyse thématique en particulier. Revue de l'Association pour la recherche qualitative, 15, 179-195.

Patriciu, S. (2001). Les représentations de l'intégration par les acteurs du système scolaire d'accueil québécois. (Thèse de doctorat inédite). Université de Sherbrooke.

Statistique Canada. (2006). La population canadienne [Internet]. www.statcan.ca/Daily/Francais. Consulté le 25/03/2007.

Tudge, J., Mokrova, I., Hatfield, B. et Karnik, R. (2009). Uses and misuses of Bronfenbrenner's bioécological theory of human development, Journal of Family Theory and Review, 1(4), 198-210.

Turnbull, A. et Turnbull, H. (1990). Families professionals, and exceptionality: A special partnership. New York : MacMillan.

Van der Maren, J.-M. (1996). Méthodes de recherche pour l'éducation (2 éd.). Montréal : Presses de l’Université de Montréal.

Alterstice-Revue Internationale de la Recherche Interculturelle, vol. 6, $n^{\circ} 1$ 
Vatz Laaroussi, M. (2007). Les relations intergénérationnelles, vecteurs de transmission et de résilience au sein des familles et réfugiées au Québec. Enfances, Familles, Générations.

Vatz Laaroussi, M. (2001). Le familial au cœur de l'immigration. Paris et Montréal : L'Harmattan.

Viens, C. (2009). Diversité et minorité : mise en œuvre de la différenciation pédagogique en milieu éducatif minoritaire francophone (Mémoire de maîtrise inédit). Université de l'Alberta. 